Pacific

Journal of

Mathematics

\title{
PANTS IMMERSED IN HYPERBOLIC 3-MANIFOLDS
}

IAN AGOL 


\title{
PANTS IMMERSED IN HYPERBOLIC 3-MANIFOLDS
}

\author{
IAN AGOL
}

\begin{abstract}
We show that a properly immersed thrice-punctured sphere in a cusped orientable hyperbolic 3-manifold is either embedded or has a single clasp in a manifold that is the Whitehead link complement or obtained by hyperbolic Dehn filling on a cusp of the Whitehead link complement.
\end{abstract}

\section{Introduction}

Let $\Sigma_{(0,3)}$ be a thrice-punctured sphere, which we will also refer to as a (pair of) pants. Adams [1985] showed that if $M$ is an oriented 3-manifold with boundary and hyperbolic interior such that $\Sigma_{(0,3)}$ embeds properly and incompressibly into $M$, then $\operatorname{int}(M)$ has an embedded totally geodesic pants. Adams also gave many examples of hyperbolic manifolds containing embedded incompressible thricepunctured spheres, and indeed one may easily produce infinite families of examples. We study properly immersed $\pi_{1}$-injective maps $f: \Sigma_{(0,3)} \rightarrow M$, where $\operatorname{int}(M)$ is hyperbolic and $f$ is not homotopic to an embedding. If one takes one component of the Whitehead link, then it bounds an immersed 2-punctured disk in the complement of the other component, which has a single clasp (double-point arc) singularity as in Figure 2. One may perform surgery on the other component to obtain infinitely many hyperbolic 3-manifolds with an immersed pants. We prove in Theorem 4.1 that this is the only possible way that a pants may be nontrivially immersed in a hyperbolic 3-manifold such that the peripheral subgroups are parabolic.

Notation. For a path metric space $X$ and a closed subspace $Y \subset X$, let $X \backslash Y$ denote the path metric completion of the open subspace $X-Y$.

\section{Parabolic $\mathrm{PSL}_{2}(\mathbb{C})$ representations of $\pi_{1}\left(\Sigma_{(0,3)}\right)$}

We will use $S=\Sigma_{(0,3)}$ from now on to indicate a pair of pants, that is, a surface of genus zero with three boundary components. Let $\partial S=c_{1} \cup c_{2} \cup c_{3}$, and let $a_{i j} \subset S$ be an embedded arc connecting $c_{i}$ to $c_{j}$ (see Figure 1). The arcs $a_{i j}$ are pairwise

MSC2000: 30F40, 57M50.

Keywords: hyperbolic geometry, topology, immersion.

The author is partially supported by NSF grant DMS-0504975 and the Guggenheim foundation. 


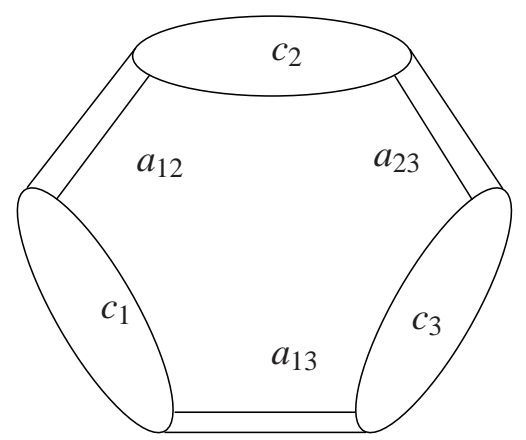

Figure 1. Labeling a pants and three seams.

disjoint when $i \neq j$, and we may assume that there is a complete hyperbolic metric on $\operatorname{int}(S)$ so that $\operatorname{int}\left(a_{i j}\right)$ is totally geodesic.

By taking a basepoint $x \in a_{12}$, the map $\left(c_{1} \cup c_{2} \cup a_{12}, x\right) \rightarrow(S, x)$ of the eyeglass graph is a homotopy equivalence. We may identify the generators of $\pi_{1}(S, x)$ with $c_{1}$ and $c_{2}$, so that the third peripheral element is $c_{1} c_{2}$, corresponding to the boundary component $c_{3}$.

There are two natural classes of representations of $\pi_{1}(S) \rightarrow \mathrm{PSL}_{2}(\mathbb{C})$ for which the peripheral elements are parabolic. The reducible representation is given by

$$
\rho\left(c_{i}\right)=\left(\begin{array}{cc}
1 & z_{i} \\
0 & 1
\end{array}\right),
$$

where we have indicated matrix lifts of the generators to $\mathrm{SL}_{2}(\mathbb{C})$. Another natural peripheral parabolic representation is given by

$$
\rho\left(c_{1}\right)=\left(\begin{array}{ll}
1 & 2 \\
0 & 1
\end{array}\right) \quad \text { and } \quad \rho\left(c_{2}\right)=\left(\begin{array}{rr}
1 & 0 \\
-2 & 1
\end{array}\right) .
$$

The following proposition shows that up, to conjugacy, these are the only peripheral parabolic representations of $\pi_{1}(S)$ (this is essentially due to Adams [1985]).

Proposition 2.1. Let $\rho: \pi_{1}(S) \rightarrow \mathrm{PSL}_{2}(\mathbb{C})$ be a representation such that the peripheral elements $\rho\left(c_{1}\right), \rho\left(c_{2}\right)$ and $\rho\left(c_{1} c_{2}\right)$ are all parabolic. Then either $\rho$ is reducible and we may conjugate $\rho$ to Equation (1), or $\rho$ is conjugate to Equation (2).

Proof. If $\rho\left(c_{1}\right)$ and $\rho\left(c_{2}\right)$ fix the same point on $\partial \mathbb{H}^{3}$, then we may send this point to $\infty$, so that the representation is conjugate to the reducible representation given above in Equation (1). Otherwise, assume that $\rho\left(c_{1}\right)$ and $\rho\left(c_{2}\right)$ fix different points on $\partial \mathbb{M}^{3}$. By a further conjugation, we may send the fixed point of $\rho\left(c_{1}\right)$ to $\infty$, and 
the fixed point of $\rho\left(c_{2}\right)$ to 0 , so that we may assume that

$$
\rho\left(c_{1}\right)=\left(\begin{array}{ll}
1 & 2 \\
0 & 1
\end{array}\right), \quad \rho\left(c_{2}\right)=\left(\begin{array}{ll}
1 & 0 \\
z & 1
\end{array}\right), \quad \rho\left(c_{1} c_{2}\right)=\left(\begin{array}{cc}
1+2 z & 2 \\
z & 1
\end{array}\right) .
$$

Since $\rho\left(c_{1} c_{2}\right)$ must be parabolic, we have $\operatorname{tr}\left(\rho\left(c_{1} c_{2}\right)\right)=2+2 z= \pm 2$, so $z=0$ or $z=-2$. The case $z=0$ means that $\rho\left(c_{2}\right)$ is trivial, so the representation is reducible, contrary to assumption. Thus, we must have $z=-2$, and we have the representation given in Equation (2).

\section{Whitehead link complement}

There is a well-known class of immersed pants in 3-manifolds that comes from drilling out a knot bounding an immersed disk with a single clasp singularity in a 3-manifold (see Figure 2). Taking a regular neighborhood of the disk with a clasp gives a solid torus. Since the boundary of the disk forms a knot inside of the solid torus, if the drilled manifold is to be hyperbolic, the torus bounding the solid torus must be compressible in the complement of the knot, and therefore bound a solid torus on the outside as well, or else bound a torus crossed with $I$. We immediately see that the only way this may happen is that we have Dehn filling on one component of the Whitehead link complement, or the Whitehead link complement itself.

In fact, we may use this immersed twice-punctured disk bounding one component of the Whitehead link to parameterize (generalized) hyperbolic Dehn filling on the other boundary component. Let $W \subset S^{3}$ be the Whitehead link, and let $M=S^{3} \backslash \backslash \mathcal{N}(W)$ be its complement. Let $\partial M=T_{1} \cup T_{2}$ be the two torus boundary components of $M$ corresponding to the two components of $W$. Let $S$ be a pants, and let $f: S \rightarrow M$ be a clasp immersion of $S$ into $M$ such that $f(\partial S) \subset T_{2}$. Suppose that we have a nonelementary representation $\rho: \pi_{1}(M) \rightarrow \mathrm{PSL}_{2}(\mathbb{C})$ such
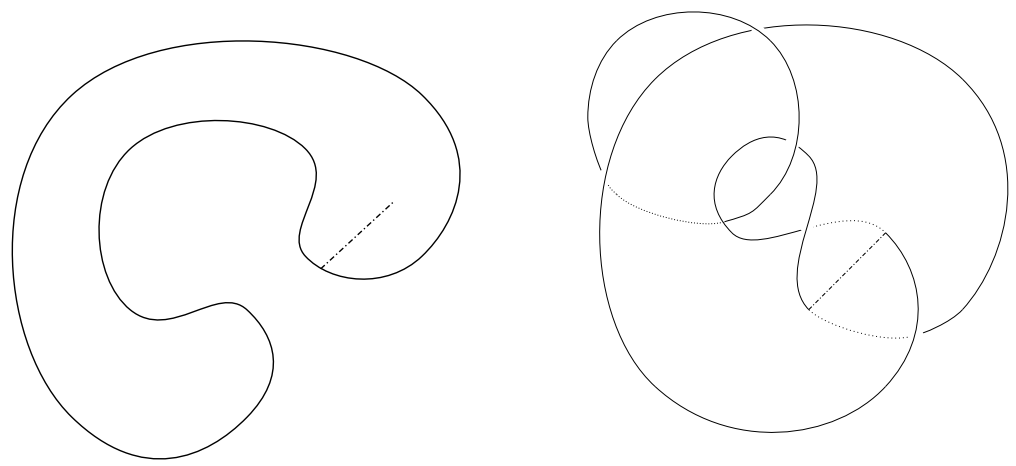

Figure 2. A twice-punctured disk in the Whitehead link complement. 


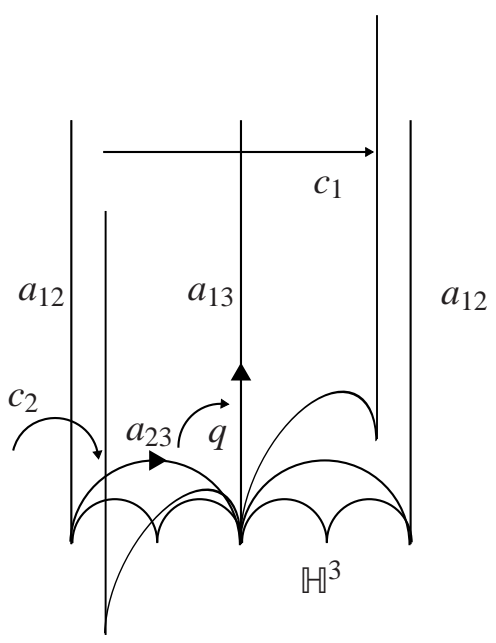

Figure 3. The universal cover of the immersion $f: S \rightarrow M$.

that $\rho\left(\pi_{1}\left(T_{2}\right)\right)<\mathrm{PSL}_{2}(\mathbb{C})$ is parabolic. Under the map $f: S \rightarrow M$ we may assume that $f\left(a_{13}\right)=f\left(a_{23}\right)$ is the set of double points of the immersion. Let $q \in \pi_{1}(M)$ be an element that sends $a_{23}$ to $a_{13}$ in such a way that $\rho(q)$ sends the fixed point of $\rho\left(c_{2}\right)$ to the fixed point of $\rho\left(c_{1} c_{2}\right)$, and the fixed point of $\rho\left(c_{1} c_{2}\right)$ to the fixed point of $\rho\left(c_{1}\right)$ (see Figure 3).

These satisfy the relations $c_{1} q^{2}=q^{2} c_{2}$ and $\left[q^{-1} c_{1} q, c_{1} c_{2}\right]=1$. One may check that $\left\{c_{1}, c_{2}, q\right\}$ generate $\pi_{1}(f(S))$, and therefore $\pi_{1}(M, x)$, because $M$ has a retraction onto the 2-complex $f(S) \cup T_{2}$, since $M \backslash \backslash f(S) \cong T_{1} \times[0,1]$ (see the discussion at the beginning of the section), and $\pi_{1}(f(S))$ generates $\pi_{1}\left(f(S) \cup T_{2}\right)$. Then $\rho\left(f_{\#}\left(\pi_{1}(S)\right)\right)$ will be a representation for which the three peripheral elements of $\pi_{1}(S)$ are parabolic. We may identify $\rho\left(f_{\#}\left(\pi_{1}(S)\right)\right)=\left\langle C_{1}, C_{2}\right\rangle$, where $C_{i}=\rho\left(f_{\#}\left(c_{i}\right)\right)$. There are two cases depending on whether this representation is reducible or not.

If $\left\langle C_{1}, C_{2}\right\rangle$ is reducible, we may assume that $\rho$ is conjugated so that this subgroup looks like Equation (1). Then $\rho(q)$ must also fix $\infty$, since $q^{-1} c_{1} q$ and $c_{1} c_{2}$ commute and therefore both fix $\infty$. In this case we see that $\rho$ is reducible.

If $\left\langle C_{1}, C_{2}\right\rangle$ is parabolic and irreducible, we may assume $\rho$ is conjugated so that this subgroup looks like Equation (2), by Proposition 2.1. Then $a_{12}$ lifts to a geodesic arc in $\mathbb{H}^{3}$ connecting 0 and $\infty, a_{13}$ connects $\infty$ and 1 , and $a_{23}$ connects 0 and 1 (see Figure 3). Keeping track of orientations, $\rho(q)(0)=1$, and $\rho(q)(1)=\infty$, which implies that

$$
\rho(q)=\left(\begin{array}{cc}
a^{-1}-a & a \\
-a & a
\end{array}\right)
$$


Thus, we see that irreducible representations of $\pi_{1}(M)$ that are parabolic on one cusp are parameterized by a single number $a \in \mathbb{C}-\{0\}$.

More importantly, the above discussion implies this intermediate result, which will be used in the proof of the main theorem:

Proposition 3.1. Let $N$ be an orientable compact 3-manifold such that $\operatorname{int}(N)$ admits a complete hyperbolic metric of finite volume. Let $f:(S, \partial S) \rightarrow(N, \partial N)$ be an essential map such that $f_{\#}\left(c_{i}\right)$ is parabolic in the hyperbolic structure on $\operatorname{int}(M)$ for each component $c_{i}$ of $\partial S$ and $f\left(a_{13}\right)=f\left(a_{23}\right)$. Then $N$ is obtained by (possibly empty) Dehn filling on one component of the Whitehead link complement.

Proof. Let $\Gamma<\mathrm{PSL}_{2}(\mathbb{C})$ be the holonomy of $\pi_{1}(N)$. Let $\left\langle C_{1}, C_{2}\right\rangle<\mathrm{PSL}_{2}(\mathbb{C})$ correspond to $f_{\#}\left(\pi_{1}(S)\right)$, where we see that $C_{i}$ and $C_{1} C_{2}$ are parabolic since $\operatorname{int}(N)$ has finite volume. If $\left\langle C_{1}, C_{2}\right\rangle$ is reducible, then $\pi_{1}(S)$ isn't essential, so we may assume that $\left\langle C_{1}, C_{2}\right\rangle$ is normalized as in Equation (2), by Proposition 2.1. Let $Q$ be an element of $\Gamma$ sending a lift of $a_{13}$ to a lift of $a_{23}$, so that $Q$ is normalized as in Equation (4). Then we see $\rho\left(\pi_{1}(M)\right)<\Gamma$, where $M$ is the Whitehead link complement and $\rho$ is the representation defined by Equations (2) and (4). Moreover, $\rho$ is parabolic on one boundary component of $M$. Since $\Gamma$ is discrete, $\rho\left(\pi_{1}(M)\right)=\Gamma^{\prime}<\Gamma$ is a finite-index subgroup. By [Francaviglia and Klaff 2006], $\operatorname{Vol}\left(\mathbb{M}^{3} / \Gamma^{\prime}\right) \leq \operatorname{Vol}(\operatorname{int}(M))=3.66 \ldots$ If $\left[\Gamma: \Gamma^{\prime}\right]>1$, then $\operatorname{Vol}(\operatorname{int}(N))=\operatorname{Vol}\left(\mathbb{M}^{3} / \Gamma\right)<1.84$, which contradicts $\operatorname{Vol}(\operatorname{int}(N)) \geq 2.0298$ by [Cao and Meyerhoff 2001]. Thus, we see that $\Gamma^{\prime}=\Gamma$, so $\Gamma$ is the discrete torsion-free homomorphic image of $\pi_{1}(M)$. In this case, we apply the analysis of [Neumann and Reid 1992] to see that every discrete irreducible homomorphic image of $\pi_{1}(M)$ in $\mathrm{PSL}_{2}(\mathbb{C})$ with one cusp remaining parabolic must come from a hyperbolic structure on the Whitehead link complement with Dehn surgery type singularity along the other boundary component. This follows from the parameterization of irreducible representations $\rho\left(\pi_{1}(M)\right)$ given in the proof of [Neumann and Reid 1992, Theorem 6.2] in terms of a single parameter $z \in \mathbb{C}-\{0\}$. Their parameter $z$ is equal to $x-x^{-1}$, where $x,-x^{-1} \in \mathbb{C}-\{0, \pm 1\}$ are the complex parameters of two pairs of tetrahedra in an ideal triangulation of $M$ corresponding to a hyperbolic structure of $\operatorname{int}(M)$ with $T_{2}$ parabolic and a Dehn surgery type singularity along $T_{1}$. The parameter $z$ must correspond to our parameter $a \neq 0$ up to a Möbius transformation, since any proper subset of $\mathbb{C}-\{0\}$ is not holomorphically equivalent to $\mathbb{C}-\{0\}$. Since $\Gamma$ is discrete and torsion-free, we conclude that $\rho\left(\pi_{1}\left(T_{1}\right)\right)$ must be a cyclic group. Therefore, the completion of $\operatorname{int}(M)$ gives a cone-manifold structure $M^{\prime}$ with cone angle an integral multiple of $2 \pi$ at the Dehn surgery type singularity along $T_{1}$ (see [Hodgson and Kerckhoff 2003] for a discussion of cone manifolds). If the cone angle is not $2 \pi$, this implies that the map $M^{\prime} \rightarrow \mathbb{H}^{3} / \Gamma$ must be a branched 
immersion that is at least two-to-one, which would imply that $\operatorname{Vol}\left(\mathbb{M}^{3} / \Gamma\right)<1.84$, giving a contradiction as before.

\section{Immersed pants}

The goal of this section is to prove the following theorem, which shows that the examples in Section 3 give the only way that a pants may be nontrivially immersed in a hyperbolic 3-manifold.

Theorem 4.1. Suppose $M$ is a connected orientable 3-manifold with boundary and interior admitting a complete hyperbolic metric. Let $S$ be a pants, and let $f:(S, \partial S) \rightarrow(M, \partial M)$ be an essential immersion such that $f_{\#}\left(c_{i}\right)$ is parabolic in the hyperbolic structure on $\operatorname{int}(M)$ for each component $c_{i}$ of $\partial S$. Then either $f$ is relatively homotopic to an embedding, or $M$ is obtained by (possibly empty) Dehn filling on one boundary component of the Whitehead link complement, and $f$ may be homotoped to have a single clasp singularity, that is, a single embedded arc of double points as in Figure 2.

Proof. We may assume that the map $f$ is homotoped so that the restriction $\left.f\right|_{\text {int }(S)}$ is totally geodesic with respect to the canonical metric on int $(M)$, by Proposition 2.1. We may identify $\operatorname{int}(M)=\mathbb{H}^{3} / \Gamma$, where $\pi_{1}(M) \cong \Gamma<\mathrm{PSL}_{2}(\mathbb{C})$ is a torsion-free discrete group. Throughout the argument, we will fix orientations on $S$ and $M$, as well as the induced orientations on $\partial S=c_{1} \cup c_{2} \cup c_{3}$ and on $\partial M$. The proof will proceed by deducing a sequence of restrictions on the manifold $M$ and the nature of the immersion $f$. We will assume from now on in the argument that the geodesic immersion $f$ is not an embedding.

Claim. $\operatorname{int}(M)$ must have finite volume.

This is achieved via an area estimate. Consider $N=\operatorname{int}(M) \backslash \backslash f(\operatorname{int}(S))$, which has a hyperbolic metric with convex boundary. For each component $N_{i}$ of $N$, there is a convex core $C\left(N_{i}\right)$ (which we take to be empty if $\chi\left(N_{i}\right) \geq 0$ ). Each $\partial C\left(N_{i}\right)$ has an intrinsic complete hyperbolic metric. If $\partial C\left(N_{i}\right)$ has a cusp, then there is some neighborhood of the cusp that is totally geodesic inside of $N_{i}$. This cusp must be parallel to a cusp of $\partial N_{i}$, which corresponds to an embedded cusp of $f(\operatorname{int}(S))$. But this cusp of $f(\operatorname{int}(S))$ has two sides, and thus there is a component $N_{j}$ on the other side of this cusp (it's possible that $j=i$ ), with a cusp of $\partial N_{j}$, and therefore of $\partial C\left(N_{j}\right)$ (which will be a distinct cusp of $\partial C\left(N_{i}\right)$ if $\left.i=j\right)$. Therefore, the cusps of $\partial C(N)$ must come in pairs, which implies that $\chi(\partial C(N))$ must be even. Throw out all components $N_{i}$ of $N$ with $C\left(N_{i}\right)=\varnothing$ to obtain $N^{\prime} \subset N$ with $\chi\left(N^{\prime}\right)<0$ if $N^{\prime} \neq \varnothing$. Then $\chi\left(\partial C\left(N^{\prime}\right)\right)<0$, and therefore $\chi\left(\partial C\left(N^{\prime}\right)\right) \leq-2$, since it is even.

There is a nearest point 1-Lipschitz retraction $N^{\prime} \rightarrow C\left(N^{\prime}\right)$, which is area decreasing when restricted to a map $\partial N^{\prime} \rightarrow \partial C\left(N^{\prime}\right)$ [Epstein and Marden 1987]. We 
have $\operatorname{Area}\left(\partial C\left(N^{\prime}\right)\right)=-2 \pi \chi\left(\partial C\left(N^{\prime}\right)\right) \geq 4 \pi$, but $4 \pi=\operatorname{Area}(\partial N) \geq \operatorname{Area}\left(\partial C\left(N^{\prime}\right)\right)$, which implies that $\operatorname{Area}\left(\partial N^{\prime}\right)=\operatorname{Area}(\partial C(N))$. This can occur if and only if $N=C\left(N^{\prime}\right)$. Since $\partial C\left(N^{\prime}\right)$ is bent along a compact measured lamination and $\partial N$ is piecewise composed of pieces of $f(S), \partial N$ must be bent along the double points of $f(S)$. But then $\partial N$ must be bent along a simple closed geodesic. This is impossible, since there are no simple closed geodesics in $S$. Thus, we have a contradiction, and we conclude that $N^{\prime}=\varnothing$. This implies that all components of $\operatorname{int}(N)$ are balls, tori, or $T^{2} \times \mathbb{R}$. This implies that $N$ has finite volume.

Claim. For each boundary component c of $\partial S, f(c)$ is embedded.

Otherwise, since $\left.f\right|_{\operatorname{int}(S)}$ is totally geodesic, $f$ would have degree at least two onto its image. Then $f$ would factor through a covering $f^{\prime}: S \rightarrow S^{\prime}$ of degree $>1$, which is impossible since $\chi(S)=-1$, so $S$ does not cover any surface $S^{\prime}$ nontrivially.

Claim. The image of $f(\partial S)$ meets at most two boundary components of $M$.

Suppose that $f(\partial S)$ meets three boundary components of $M$. Then each component of $\partial S$ maps to a distinct boundary component of $M$. Thus $0 \neq f_{*}[c] \in H_{1}(\partial M)$ for each component $c \subset \partial S$, and therefore $0 \neq f_{*}([S]) \in H_{2}(M, \partial M)$. Since $\operatorname{int}(M)$ is hyperbolic, each homology class of $H_{2}(M, \partial M)$ has Thurston norm $\geq 1$ [Thurston 1986]. There exists an embedded orientable incompressible surface $\Sigma \subset M$ such that $f_{*}([S])=[\Sigma] \in H_{2}(M, \partial M)$ by [Gabai 1983], and such that $\chi_{-}(\Sigma)=\chi_{-}(S)=1$, which implies that $\Sigma$ is connected and $\chi(\Sigma)=-1$. Since each boundary component of $S$ goes to a different boundary component of $M$ under the map $f$, we see that $\Sigma$ is an embedded pants such that $\operatorname{int}(\Sigma)$ is totally geodesic. Also, each component of $\partial \Sigma$ is parallel to a component of $f(\partial S)$, since each component of $f(\partial S)$ is homologous to a component of $\partial \Sigma$. Since $f$ and $\Sigma$ are both totally geodesic, the boundary components $f(\partial S) \cap \partial \Sigma=\varnothing$, since otherwise we would have $f(S)=\Sigma$, which contradicts the assumption that $f$ is not embedded. Then $f^{-1}(\Sigma)$ is a collection of embedded geodesic curves, since $\Sigma$ is embedded. But these curves miss $\partial S$, which means $f^{-1}(\Sigma)=\varnothing$, since there are no closed embedded geodesics in $\operatorname{int}(S)$. But this is also a contradiction, since $M \backslash f(S)$ is a union of regions with abelian fundamental group, and thus cannot contain the embedded pants $\Sigma$.

Claim. The image of $f(\partial S)$ meets only one boundary component of $M$.

Suppose that $f(\partial S) \subset T_{1} \cup T_{2} \subset \partial M$, where $T_{i}$ is a torus. Let $\partial S=c_{1} \cup c_{2} \cup c_{3}$. Then we may assume that $f\left(c_{1} \cup c_{2}\right) \subset T_{1}$ and $f\left(c_{3}\right) \subset T_{2}$, since we are assuming for contradiction that $f(\partial S)$ is not contained in a single boundary component. This implies that $0 \neq f_{*}\left(\left[c_{3}\right]\right) \in H_{1}\left(T_{2}\right)$, and thus $0 \neq f_{*}([S]) \in H_{2}(M, \partial M)$. Suppose $\Sigma \subset M$ be an embedded orientable incompressible surface satisfying 
$[\Sigma]=f_{*}([S]) \in H_{2}(M, \partial M)$ and $\chi_{-}(\Sigma)=1$. Then $\Sigma$ is either a pants or a punctured torus. First assume that $\Sigma$ is a pants. Then we may assume that $\operatorname{int}(\Sigma)$ is totally geodesic in $\operatorname{int}(M)$. Let $\partial \Sigma=c_{1}^{\prime} \cup c_{2}^{\prime} \cup c_{3}^{\prime}$, where $c_{3}^{\prime} \subset T_{2}$ is a curve parallel to $f\left(c_{3}\right)$, which must be disjoint since $f(S)$ and $\Sigma$ do not coincide. Then $\gamma=f^{-1}(\Sigma) \subset S$ is a (possibly disconnected) embedded 1-manifold with geodesic interior, which must be disjoint from $c_{3}$. Since $c_{1}^{\prime}$ and $c_{2}^{\prime}$ are parallel, and $\left[c_{1}^{\prime}\right]+\left[c_{2}^{\prime}\right]=f_{*}\left(\left[c_{1}\right]+\left[c_{2}\right]\right) \in H_{1}\left(T_{1}\right)$, either $f\left(c_{i}\right) \cap \partial \Sigma=\varnothing$ for both $i=1,2$, or $\left|f\left(c_{i}\right) \cap \partial \Sigma\right| \geq 2$. In the first case, we obtain a contradiction as before in the case that we assumed that $f(\partial S)$ meets three boundary components of $M$. In the second case, we have a contradiction, since there is no embedded curve with geodesic interior $\gamma \subset S$ such that $\partial \gamma \subset c_{1} \cup c_{2}$ and $\left|\gamma \cap c_{i}\right| \geq 2$ for $i=1,2$.

Thus, we must be in the case that $\Sigma$ is a punctured torus. We may assume that $\Sigma$ is quasifuchsian, that is, $\Sigma$ has no accidental parabolics; otherwise there would be an embedded pants homologous to $\Sigma$, giving a contradiction as before. We may assume that $f\left(c_{3}\right) \cap \partial \Sigma=\varnothing$, by an isotopy, since these curves are homologically parallel and therefore isotopic in $\partial M$. We have $f^{-1}(\Sigma)$ is an embedded union of curves on $S$ that miss $\partial S$. Homotope $f$ so that the number of components of $f^{-1}(\Sigma)$ has minimal cardinality. We may assume that each component of $f^{-1}(\Sigma)$ is essential in $\Sigma$, since otherwise a homotopy would reduce the cardinality of $\left|f^{-1}(\Sigma)\right|$. Therefore if $f^{-1}(\Sigma)$ is nonempty, each component of $f^{-1}(\Sigma)$ is an embedded closed curve that is boundary parallel in $S$. An outermost such curve $c$ on $S$ cobounds an annulus $A$ with a boundary component $c_{i}$ of $S$. Then $f(c)$ represents a parabolic element in $\Sigma$, and therefore must be boundary parallel in $\Sigma$ since $\Sigma$ has no accidental parabolics. Thus, since $M$ is acylindrical, we may homotope $f$ to reduce the number of components of $f^{-1}(\Sigma)$, a contradiction. If $f^{-1}(\Sigma)$ is empty, we obtain a contradiction as before. Thus, we conclude that $f(\partial S)$ meets at most one boundary component of $M$.

Claim. $f(\partial S)$ is not embedded.

For sake of contradiction, assume that $f(\partial S) \subset \partial M$ is embedded. In this case, again we have $0 \neq f_{*}([\partial S]) \in H_{1}(\partial M)$, so we have $0 \neq f_{*}([S]) \in H_{2}(M, \partial M)$. Thus, there is an embedded incompressible norm-minimizing surface $\Sigma \subset M$ such that $\chi_{-}(\Sigma)=1$ and $f_{*}([S])=[\Sigma] \in H_{2}(M, \partial M)$. If $\Sigma$ is a pants, then $\partial \Sigma$ must be parallel to $f(\partial S)$ since they are homologically parallel. We obtain a contradiction as before in the case that $M$ had three boundary components. If $\Sigma$ is a punctured torus with no accidental parabolic, then $f^{-1}(\Sigma) \subset S$ is a collection of embedded closed curves, which are therefore boundary parallel. As in the previous paragraph, we homotope $f$ so that $f^{-1}(\Sigma)$ has fewer components, until $f^{-1}(\Sigma)$ is empty, which gives a contradiction as before, proving that $f(\partial S)$ is not embedded. 
We may now assume that $f(\partial S)$ meets only one boundary component of $M$, and that the curves $f\left(c_{i}\right)$ are not all parallel in $\partial M$. We introduce some further notation now to be used throughout the rest of the proof. Let $H \subset \operatorname{int}(M)$ be a maximal open horocusp containing neighborhoods of the ends of $f(\operatorname{int}(S))$ (and thus homeomorphic to $\left.T^{2} \times \mathbb{R}\right)$. Then $\bar{H} \subset \operatorname{int}(M)$ will have self-tangencies. The preimage of $H$ in $\mathbb{Q}^{3}$ is a family of horoballs invariant under the action of $\Gamma \cong \pi_{1}(M)$. The preimage $f^{-1}(H) \subset \operatorname{int}(S)$ contains a collection of horocycles in $\operatorname{int}(S)$ surrounding $\partial S$. There are three curves $h_{i} \rightarrow \operatorname{int}(S)$ such that $\bigcup_{i=1}^{3} h_{i} \subset f^{-1}(\partial \bar{H})$ and such that $h_{i}$ is homotopic to $c_{i}$ in $S$. The curves $h_{i}$ might not be embedded, since they may have self-tangencies mapping to the self-tangent points of $\bar{H}$ under the map $f$. The curves $f\left(h_{i}\right) \subset \partial \bar{H}$ are geodesics in the intrinsic euclidean metric on $\partial \bar{H}$.

Claim. For $i \neq j$, we have $l\left(h_{i}\right) l\left(h_{j}\right) \leq 4$.

This may be shown by a simple computation in hyperbolic geometry. One way to see this is to expand $h_{i}$ and $h_{j}$ keeping them horocycles, until they become tangent horocycles $h_{i}^{\prime}, h_{j}^{\prime}$. Then $l\left(h_{i}^{\prime}\right) l\left(h_{j}^{\prime}\right)=4$. This may be shown by shrinking the longer of the two cycles, while expanding the smaller, until we obtain two tangent horocycles with the same length. This operation preserves the product of the lengths, since if we shrink a distance $d$, then the length gets multiplied by $e^{-d}$, while the expanded horocycle has length multiplied by $e^{d}$, keeping the product constant. Once we reach equal size tangent horocycles, both have length 2 , so the product is 4 . This horocycle bound is special to pants.

Let $\Delta: H_{1}(T) \times H_{1}(T) \rightarrow \mathbb{Z}$ be the algebraic intersection number of a torus $T$. We will use the notation $\Delta(a, b)=\Delta([a],[b])$ for embedded oriented curves $a, b \subset T$.

Claim. $\left|\Delta\left(f\left(c_{i}\right), f\left(c_{j}\right)\right)\right| \leq 1$ for all $i, j$.

If $f\left(h_{i}\right)$ and $f\left(h_{j}\right)$ are horocycles in $\partial \bar{H}$, then

$$
\left|\Delta\left(f\left(h_{i}\right), f\left(h_{j}\right)\right)\right| \operatorname{Area}(\partial \bar{H}) \leq l\left(h_{i}\right) \cdot l\left(h_{j}\right) .
$$

This implies that for any $i \neq j$, if $\left|\Delta\left(f\left(h_{i}\right), f\left(h_{j}\right)\right)\right|>1$, then $\operatorname{Area}(\partial \bar{H}) \leq 2$. But this is a contradiction by [Cao and Meyerhoff 2001, Proposition 5.8], which states that the area of $\partial \bar{H}$ is $\geq 3.35$. Thus, we conclude that $\left|\Delta\left(f\left(c_{i}\right), f\left(c_{j}\right)\right)\right| \leq 1$ for all $i, j$.

Claim. We may assume that $0 \neq f_{*}([\partial S]) \in H_{1}(\partial M)$.

Suppose that $f([\partial S])=0 \in H_{1}(\partial M)$, so that $\left[f\left(c_{3}\right)\right]=-\left[f\left(c_{1}\right)\right]-\left[f\left(c_{2}\right)\right]$ (with appropriately chosen orientations). Then, since $\Delta$ is bilinear and skew-symmetric,

$$
\begin{aligned}
& \Delta\left(f\left(c_{1}\right), f\left(c_{2}\right)\right)+\Delta\left(f\left(c_{1}\right), f\left(c_{3}\right)\right)+\Delta\left(f\left(c_{2}\right), f\left(c_{3}\right)\right)= \\
& \Delta\left(f\left(c_{1}\right), f\left(c_{2}\right)\right)+\Delta\left(f\left(c_{1}\right)+f\left(c_{2}\right),-f\left(c_{1}\right)-f\left(c_{2}\right)\right)=\Delta\left(f\left(c_{1}\right), f\left(c_{2}\right)\right) .
\end{aligned}
$$


But we also have that $\left|\Delta\left(f\left(c_{1}\right), f\left(c_{2}\right)\right)\right|+\left|\Delta\left(f\left(c_{1}\right), f\left(c_{3}\right)\right)\right|+\left|\Delta\left(f\left(c_{2}\right), f\left(c_{3}\right)\right)\right|$ represents the number of endpoints of the arcs of self-intersection of the map $f: S \rightarrow M$, and therefore must be even. Therefore the term at (5) is also even, since it has the same parity. Thus, we see that $\Delta\left(f\left(c_{1}\right), f\left(c_{2}\right)\right)$ must be even, which implies that $\Delta\left(f\left(c_{1}\right), f\left(c_{3}\right)\right)$ and $\Delta\left(f\left(c_{2}\right), f\left(c_{3}\right)\right)$ are also even, by symmetry of the indices. Since $\Delta\left(f\left(c_{i}\right), f\left(c_{j}\right)\right) \neq 0$ for some $i, j$ because $f(\partial S)$ is not embedded, we must have $\left|\Delta\left(f\left(c_{i}\right), f\left(c_{j}\right)\right)\right|=2$, which contradicts the previous claim and thus implies that $0 \neq f_{*}([\partial S]) \in H_{1}(\partial M)$.

Since $\left|\Delta\left(f\left(c_{i}\right), f\left(c_{j}\right)\right)\right| \leq 1$, and the total parity of the 3 intersections is even, we conclude that $\Delta\left(f\left(c_{i}\right), f\left(c_{j}\right)\right)=0$ for some $i \neq j$, that is, $f\left(c_{i}\right)$ and $f\left(c_{j}\right)$ must be parallel (or antiparallel, keeping track of orientations). Since not all three are parallel, we may assume that $f\left(c_{1}\right)$ and $f\left(c_{2}\right)$ are parallel, and $f\left(c_{3}\right)$ intersects both precisely once.and Thus, we have $l\left(f\left(h_{1}\right)\right)=l\left(f\left(h_{2}\right)\right)=b$ and $l\left(f\left(h_{3}\right)\right)=a$ for some $a, b$ such that $a b \leq 4$. Moreover, $1 \leq b \leq 2$, where the lower bound follows from the fact that the length of a horocycle in a maximal cusp is at least 1 [Adams 2002], and the upper bound follows from the facts that $l\left(h_{1}\right)=l\left(h_{2}\right)=b$ and $l\left(h_{1}\right) l\left(h_{2}\right) \leq 4$. Clearly we also have $a \leq 4$.

In $S$, there are embedded essential arcs (unique up to isotopy) $a_{i j}$ connecting $c_{i}$ to $c_{j}$. We may assume that $\operatorname{int}\left(a_{i j}\right) \subset \operatorname{int}(S)$ is a geodesic. We may use these arcs to analyze the preimage $\widetilde{H} \subset \mathbb{M}^{3}$ of the horoball neighborhood of the cusp $H \subset \operatorname{int}(M)$. Identifying $\mathbb{\boxplus}^{3}$ with the upper half space model, we may conjugate $\Gamma$ so that there is a component of $\widetilde{H}$ which is a horoball $H_{\infty} \subset \mathbb{H}^{3}$ centered at $\infty$, so that the boundary of $H_{\infty}$ is a Euclidean plane at height 1, and so that the intrinsic hyperbolic metric on $\partial H_{\infty}$ is the same as the induced Euclidean metric. Then $\widetilde{H}=\bigcup_{p \in \Gamma(\infty)} H_{p}$, where $H_{p}$ is a horoball component of $\widetilde{H}$ centered at $p \in \partial \llbracket^{3}$. Up to the stabilizer of $H_{\infty}$, there are two lifts of each geodesic $\operatorname{arc} \operatorname{int}\left(a_{i j}\right)$ to $\mathbb{T}^{3}$ with one endpoint at $\infty$, corresponding to the two ends of $a_{i j}$. If $f\left(a_{13}\right) \neq f\left(a_{23}\right)$, then we see four horoballs from infinity of height $a b / 4$ up to the stabilizer of $H_{\infty}$ corresponding to the four distinct lifts of these two arcs, where $a=l\left(h_{3}\right)$ and $b=l\left(h_{1}\right)$. By possibly conjugating $\Gamma$, we may assume that the four horoballs are centered at $0, a / 2, w_{1}$, and $w_{2}$, where $w_{1}, w_{2} \in \mathbb{C}$.

Claim. If $f\left(a_{13}\right) \neq f\left(a_{23}\right)$, then $a b<4$.

If $f\left(a_{13}\right) \neq f\left(a_{23}\right)$ and $a b=4$, then these four distinct horoballs (up to the stabilizer of $\infty$ ) have height 1 . We show that this gives a contradiction. First, we will consider the case that $a<4$ and $b<2$. We get three strings of tangent horoballs up to the stabilizer of $\infty$ corresponding to each cusp of $S$. One string consists of height 1 horoballs $H_{k a / 2}$ and horoballs $H_{k a / 2+a / 4}$ of height $a^{2} / 16$ for $k \in \mathbb{Z}$. The other pair of strings has height 1 horoballs $H_{k u+w_{i}}$, where $|u|=b$, $u \in \mathbb{C}-\mathbb{R}, \quad k \in \mathbb{Z}$, and $w_{1}, w_{2} \in \mathbb{C}$, and horoballs $H_{(k+1 / 2) u+w_{i}}$ of height $b^{2} / 4$. 


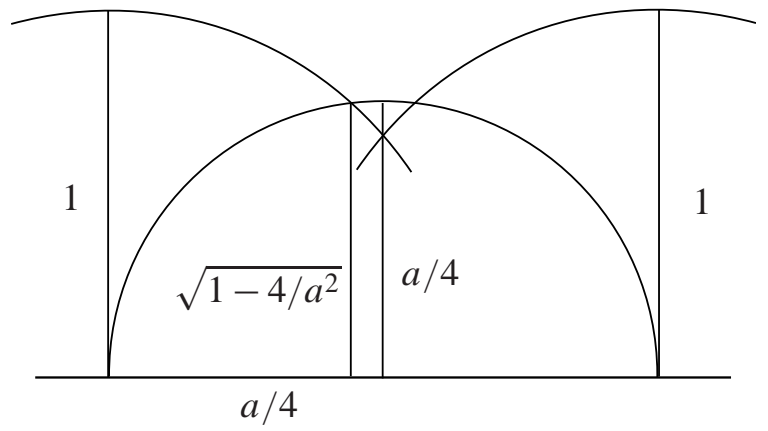

Figure 4. The imaginary part of $k u+w_{i}$ is greater in absolute value than $\sqrt{1-4 / a^{2}}$ when $a \geq 2 \sqrt{2}$.

Then the horoballs $H_{k u+w_{i}}$ must each be disjoint from the horoballs $H_{k a / 2}$ since $f\left(a_{13}\right) \neq f\left(a_{23}\right)$, and disjoint from the horoballs $H_{k a / 2+a / 4}$ since they have distinct heights (since $a<4$ by assumption). This implies that $\left|k u+w_{i}-k^{\prime} a / 2\right| \geq 1$ and $\left|k u+w_{i}-\left(k^{\prime} a / 2+a / 4\right)\right| \geq a / 4$ for all $k, k^{\prime} \in \mathbb{Z}$. A geometric computation shows that the only points satisfying this property must be distance $\sqrt{1-4 / a^{2}}$ from the real axis (see Figure 4) when $a \geq 2 \sqrt{2}$, and distance $\sqrt{1-(a / 4)^{2}}$ when $a \leq 2 \sqrt{2}$. The horoballs $H_{(k-1) u+w_{i}}$ and $H_{k u+w_{i}}$ must straddle the real axis (and therefore the strings of $H_{k^{\prime} a / 4}$-balls) for some $k \in \mathbb{Z}$, and therefore must be separated by $2 \sqrt{1-4 / a^{2}}$ when $a \geq 2 \sqrt{2}$. Since $b=4 / a$, we see that $b=4 / a \geq 2 \sqrt{1-4 / a^{2}}$, so $a \leq 2 \sqrt{2}$, thus $a=2 \sqrt{2}$. Similarly, we see that if $a \leq 2 \sqrt{2}$, then $b \geq \sqrt{2}$. Exchanging the roles of $a / 2$ and $b$ in the above argument, we see that $H_{(k-1) a / 2}$ and $H_{k a / 2}$ must straddle $u \mathbb{R}+w_{i}$ for some $k$. As before, we get $b \leq \sqrt{2}$ (where we are assuming $b<2$ to get $H_{k a / 2}$ disjoint from $H_{\left(k^{\prime}+1 / 2\right) u+w_{i}}$ ). But this implies in either case that $a=2 \sqrt{2}, b=\sqrt{2}$, and $w=\sqrt{2} / 2+\sqrt{2} / 2 i$. Therefore the horoballs of height $1 / 2$ in the two strings must coincide. This implies that the arcs $a_{12}$ and $a_{33}$ (the arc connecting $c_{3}$ to itself) in $S$ must get identified by $f(S)$, which is impossible since there would be an isometry fixing a lift of $f\left(a_{12} \cap a_{33}\right) \in \mathbb{H}^{3}$. In fact, this configuration of horoballs does occur in the Whitehead link complement, but not corresponding to immersed pants in the manner hypothesized.

We now turn to the case that $a=4$. Then we have $b=1$, and this implies that $M$ is the figure eight knot complement by [Adams 2002]. Then $M$ satisfies the conclusion of the theorem, but we need to show that the immersed pants in $M$ are of the claimed type, and in fact we show that this case does not occur. The previous argument goes through if $f\left(a_{i 3}\right) \neq f\left(a_{33}\right)$ for $i=1$ or $i=2$, since this is equivalent to $k a / 2+a / 4 \neq k^{\prime} u+w_{i}$ for $k, k^{\prime} \in \mathbb{Z}$. So assuming that $f\left(a_{13}\right)=f\left(a_{33}\right)$ and $f\left(a_{23}\right)=f\left(a_{33}\right)$, we contradict the assumption of the claim. 
Finally, we consider $b=2$ and therefore $a=2$. Again, the argument above works if $k a / 4 \neq\left(k^{\prime}+1 / 2\right) u+w_{i}$ for all $k, k^{\prime} \in \mathbb{Z}$, which is equivalent to $f\left(a_{i 3}\right) \neq f\left(a_{12}\right)$ for $i=1,2$. So we must have $f\left(a_{12}\right)=f\left(a_{13}\right)=f\left(a_{23}\right)$, contradicting the assumption of the claim.

Claim. $f\left(a_{13}\right)=f\left(a_{23}\right)$.

For contradiction, assume that $f\left(a_{13}\right) \neq f\left(a_{23}\right)$. Thus, we may assume that $a b<4$. In this case, we have two full-sized horoballs [Adams 1987], and four horoballs of height $a b / 4$ up to the action of the stabilizer in $\Gamma$ of $\infty$. We may estimate the "seen area" of these horoballs, as in [Cao and Meyerhoff 2001]. The two fullsized horoballs each have radius $1 / 2$, so they contribute an area of $\pi / 2$. The other four balls may be "overshadowed" by the full-sized balls, but the distance of the centers in $\partial \mathbb{H}^{3}$ must be at least $\sqrt{a b / 4}$ from the centers of the full-sized balls (of radius $1 / 2)$. Thus, disks of radius $\sqrt{a b / 4}-1 / 2$ will be embedded when centered at the centers of the horoballs. Thus we see an area of $\pi / 2+4 \pi(\sqrt{a b} / 2-1 / 2)^{2}$. But we have $\operatorname{Area}(\partial \bar{H}) \leq a b$. So we have

$$
\pi / 2+4 \pi(\sqrt{a b} / 2-1 / 2)^{2} \leq a b .
$$

This is a quadratic inequality in $\sqrt{a b}$, and we complete the square to see that there are no positive solutions $\sqrt{a b}$ satisfying the inequality.

This implies that we must have $f\left(a_{13}\right)=f\left(a_{23}\right)$. In this case, if $a_{i 3}$ is oriented away from $c_{3}$, then the orientations of $f\left(a_{13}\right)$ and $f\left(a_{23}\right)$ must be reversed under this identification (otherwise the two arcs would be identified by a parabolic translation, which is impossible because $f\left(c_{3}\right)$ is embedded). By Proposition 3.1, we see that $M$ is obtained by Dehn filling on one component of the Whitehead link complement.

\section{Conclusion}

The result in this paper answers a special case of the general question, How singular can an immersed surface be? One may be able to extend the results in this paper to understand immersed twice-punctured two-sided projective planes in nonorientable hyperbolic 3-manifolds. These are totally geodesic for the same reason that pants are, and it is likely that one could classify all the nonembedded immersions. It is likely that one could also give a classification of collections of pants in a hyperbolic 3-manifold. That is, one could classify the patterns of intersections that may arise. It's also likely possible to classify $\pi_{1}$-injective immersions of punctured tori, and maybe some other simple surfaces, into hyperbolic 3-manifolds. It would be interesting to extend the classification of immersed pants to arbitrary 3-manifolds by analyzing how the surface cuts through the various 
pieces of the geometric decomposition. For turnovers immersed in hyperbolic 3orbifolds, G. Martin [1996] showed that a $(2,3, p)$-triangle group in a hyperbolic 3 -orbifold is embedded for $p \geq 7$. However, there are other triangle groups that are immersed in 3-orbifolds; see [Maclachlan 1996; Rafalski 2007].

For arbitrary surfaces, it's hard to imagine a complete classification of immersions into 3-manifolds. We conjecture a structural result for immersed surfaces: For a surface of fixed topological type, there are finitely many homeomorphism types of 3-manifolds and $\pi_{1}$-injective maps of the surface into these manifolds such that any $\pi_{1}$-injective immersion of the surface into a 3-manifold factors through an embedding of one of these manifolds. This conjecture seems feasible at least when the target manifold is hyperbolic, and the main result in this paper proves this conjecture for immersions of pants. Rafalski [2007] has shown the analogue of this conjecture for turnovers immersed in hyperbolic 3-orbifolds.

\section{Acknowledgments}

I thank Shawn Rafalski for suggesting some simplifications to the argument, and the referee for helpful suggestions.

\section{References}

[Adams 1985] C. C. Adams, "Thrice-punctured spheres in hyperbolic 3-manifolds", Trans. Amer. Math. Soc. 287:2 (1985), 645-656. MR 86k:57008 Zbl 0527.57002

[Adams 1987] C. C. Adams, "The noncompact hyperbolic 3-manifold of minimal volume", Proc. Amer. Math. Soc. 100:4 (1987), 601-606. MR 88m:57018 Zbl 0634.57008

[Adams 2002] C. C. Adams, "Waist size for cusps in hyperbolic 3-manifolds", Topology 41:2 (2002), 257-270. MR 2003e:57023 Zbl 0985.57012

[Cao and Meyerhoff 2001] C. Cao and G. R. Meyerhoff, "The orientable cusped hyperbolic 3manifolds of minimum volume", Invent. Math. 146:3 (2001), 451-478. MR 2002i:57016 Zbl 1028.57010

[Epstein and Marden 1987] D. B. A. Epstein and A. Marden, "Convex hulls in hyperbolic space, a theorem of Sullivan, and measured pleated surfaces", pp. 113-253 in Analytical and geometric aspects of hyperbolic space (Coventry/Durham, 1984), edited by D. B. A. Epstein, London Math. Soc. Lecture Note Ser. 111, Cambridge Univ. Press, 1987. MR 89c:52014 Zbl 0612.57010

[Francaviglia and Klaff 2006] S. Francaviglia and B. Klaff, "Maximal volume representations are Fuchsian”, Geom. Dedicata 117 (2006), 111-124. MR 2007d:51019 Zbl 1096.51004

[Gabai 1983] D. Gabai, "Foliations and the topology of 3-manifolds", J. Differential Geom. 18:3 (1983), 445-503. MR 86a:57009 Zbl 0533.57013

[Hodgson and Kerckhoff 2003] C. D. Hodgson and S. P. Kerckhoff, "Harmonic deformations of hyperbolic 3-manifolds", pp. 41-73 in Kleinian groups and hyperbolic 3-manifolds (Warwick, 2001), edited by Y. Komori et al., London Math. Soc. Lecture Note Ser. 299, Cambridge Univ. Press, 2003. MR 2005d:30066 Zbl 1051.57018

[Maclachlan 1996] C. Maclachlan, "Triangle subgroups of hyperbolic tetrahedral groups", Pacific J. Math. 176:1 (1996), 195-203. MR 98d:20056 Zbl 0865.20031 
[Martin 1996] G. J. Martin, “Triangle subgroups of Kleinian groups”, Comment. Math. Helv. 71:3 (1996), 339-361. MR 97g:57016 Zbl 0865.30059

[Neumann and Reid 1992] W. D. Neumann and A. W. Reid, "Arithmetic of hyperbolic manifolds", pp. 273-310 in Topology '90 (Columbus, OH, 1990), edited by B. Apanasov et al., Ohio State Univ. Math. Res. Inst. Publ. 1, de Gruyter, Berlin, 1992. MR 94c:57024 Zbl 0777.57007

[Rafalski 2007] S. Rafalski, "Immersed turnovers in hyperbolic 3-orbifolds", preprint, 2007. arXiv $0708.3415 \mathrm{v} 1$

[Thurston 1986] W. P. Thurston, "A norm for the homology of 3-manifolds", Mem. Amer. Math. Soc. 59:339 (1986), i-vi and 99-130. MR 88h:57014 Zbl 0585.57006

Received February 19, 2008.

IAN AGOL

UNIVERSITY OF CALIFORNIA

970 EVANS HaLL \#3840

BERKELEY, CA 94720-3840

UNITED STATES

ianagol@math.berkeley.edu

http://math.berkeley.edu/ ianagol/ 